\section{Letter to} the Editor

\title{
The Use of Subvalvular Repair for Functional Mitral Regurgitation
}

\author{
Francesco Nappi, MD
}

Keywords: functional mitral regurgitation, subvalvular repair, restrictive mitral annuloplasty, papillary muscle approximation

Furukawa and colleagues ${ }^{1)}$ reported their experience of customized mitral valve repair for functional mitral regurgitation (FMR) in patients with low left ventricular ejection fraction (LVEF) alongside implications for preoperative right ventricular (RV) function. In all, 14 patients with non-advanced left ventricular (LV) dimensions remodeling underwent restrictive mitral annuloplasty (RMA) using an undersized (1-2 sizes) semi-rigid full ring in 12 patients and a partially flexible ring in the remaining two. In total, 22 patients at risk of recurrent mitral regurgitation (MR) due to advanced remodeling of LV dimensions underwent an additional subvalvular repair (SVR) with RMA. SVR was performed using both papillary muscle approximation (PMA $n=11$ ) and papillary muscle relocation (PMR $n=6$ ). The other surgical procedures used were left ventricular reconstruction $($ LVR $n=6)$ and secondary chord cutting $(\mathrm{SCC}, \mathrm{n}=4)$. The parameters to determine the advanced remodeled LVs were as follows: LV end-diastolic dimension (LVDd) $>65 \mathrm{~mm}, \mathrm{LV}$ end-systolic dimension (LVDs) $>51 \mathrm{~mm}$, coaptation tenting height $(\mathrm{TH}) \geq 11 \mathrm{~mm}$, posterior mitral leaflet angle (PLA) $>45^{\circ}$, and the seagull

Department of Cardiac Surgery, Centre Cardiologique du Nord de Saint-Denis, Paris, France

Received: July 7, 2020; Accepted: July 7, 2020

Corresponding author: Francesco Nappi, MD. Department of Cardiac Surgery, Centre Cardiologique du Nord de Saint-Denis (CCN), 36 Rue des Moulins Gémeaux,93200 Saint-Denis, France Email: francesconappi2@gmail.com

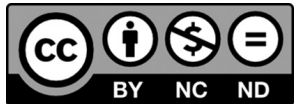

This work is licensed under a Creative Commons Attribution-NonCommercialNoDerivatives International License.

(C)2021 The Editorial Committee of Annals of Thoracic and Cardiovascular Surgery sign. ${ }^{2,3)}$ Importantly, the authors assessed the right ventricular fractional area change (RVFAC) to establish if the RV function was associated with cardiac-related mortality. ${ }^{1)}$

\section{Subvalvular Procedures}

Subvalvular procedures are commonly used to complement annuloplasty to recondition both the functionality and configuration of the subvalvular apparatus. The main effect of SVR is the reduction of tethering forces exerted on both leaflets of mitral valve (MV) due to lateral and posterior dislocation of PMs..-6) Bothe et al. ${ }^{7)}$ suggested that the displacement of the posterior papillary muscle along the posterolateral vector is the main pathophysiological mechanism causing apical leaflet tethering during ischemic mitral regurgitation (IMR), thereby leading to the identification of only two vectors. The first is the posterolateral vector which develops in the conditions of ischemia/necrosis of the posterior and/or inferior myocardial wall when the posteromedial papillary muscle (PMPM) is involved. The second is represented by the lateral vector that occurs with the displacement of the anterolateral papillary muscle (ALPM) during the necrosis/ischemia of anterior and/or lateral myocardial wall.

\section{Papillary Muscle Approximation}

The goal of papillary muscle interventions is to reestablish the correct LV geometry which was compromised by the vector displacement of the PMs. In IMR, this geometric alteration should be considered in three dimensions: the anteroposterior diameter of the annulus, the tenting area, and the interpapillary muscle distance., ${ }^{2,46}$ We use 4-0 Goretex sutures with pledgets for the types of PMs anatomically classified as I, II, and III and a 4-0 Goretex 
prosthesis for types IV and V.. ${ }^{4-68)}$ Furukawa et al. ${ }^{1)}$ reported 11 patients who underwent PMA but did not describe the anatomy of PMs. In addition, the authors used PMA with concurrent LVR by performing an approximation of the PM from the base to the center with two mattress sutures (3-0 polypropylene) and through the LV incision. We can spontaneously to deduce that the anatomical classification of PM corresponded to type I, II, or III.

\section{Clinical evidence}

In PMA randomized clinical trial, ${ }^{5}$ we evaluated the effect on PMA on long-term outcomes in 96 patients who had moderate-to-severe MR. The 48 patients undergoing combined SVR and RMA a similar 5-year survival rate than those $(n=48)$ of the RMA only group (22.9\% vs. $29.2 \%$; hazard ratio [HR] 0.76 ; $95 \%$ confidence interval $[\mathrm{CI}], 0.35-1.68, \mathrm{P}=0.502$ ). Concerning the experience of moderate-to-severe MR recurrence, although no statistically significant difference between the two groups was reported after 2 years (RMA $13.2 \%$ vs PMA $15.0 \%$ ), at 5 years, we noted a trending difference between the proportion of surviving patients with recurrence of severe MR (restrictive anuloplasty [RA] $23.5 \%$ vs. PMA $10.8 \%, \mathrm{P}=0.153$ ). At 5-year follow-up after surgery in recipients of RMA combined to SVR there was a trending increase in further re-hospitalization for heart failure that were lower to those who had received RMA alone (23.8\% vs 38\%, $\mathrm{P}=0.136)$. During a follow-up period of 5 years, the incidence of major adverse cardiac or cerebrovascular event was significantly reduced in the PMA group in the last year of follow-up (HR: 0.10 ; $95 \%$ CI: 0.02 to $0.49, \mathrm{P}=0.004$ ).

Furukawa et al. ${ }^{1)}$ revealed no difference in a 3-year rate of freedom from cardiac-related mortality when comparing the patients who received RMA alone to those who were managed with the combined RMA and SVR (93\% vs $81 \% ; \mathrm{P}=0.3$ ). As highlighted in the results, although one patient who underwent an isolated RMA experienced recurrence of MR $\geq 2$; however, LVDs significantly decreased (from $46 \pm 8$ to $37 \pm 12 \mathrm{~mm}$; P $<0.01$ ) and LVEF significantly improved (from $29 \pm 7 \%$ to $46 \pm 18 \% ; \mathrm{P}=0.01$ ). Higher improvement rates for LVDd, LVDs, LAD, and LVF have been documented when the SVR was associated with RMA. At 5-year follow-up, LVDd and LVDs significantly decreased (from $64 \pm 7$ to $59 \pm 9 \mathrm{~mm} ; \mathrm{P}<0.01$ and $56 \pm 8$ to $49 \pm 11 \mathrm{~mm}$; $\mathrm{P}<0.01)$. The same favorable results were noted for the decreasing of LVD values (from $44 \pm 8$ to $41 \pm 5 \mathrm{~mm}$; $\mathrm{P}=0.03$ ) and for the LVEF values that significantly improved (from $27 \pm 8 \%$ to $36 \pm 14 \% ; \mathrm{P}=0.02$ ). Note that three patients $(14 \%)$ who received the combined RMA and SVR, had recurrence of MR grade $\geq 2$ with one patient required a mitral valve replacement 56 months after the first surgery. In patients with combined surgery, the 3-year and 5-year results showed safety and effectiveness of procedure with non-recurrence rates of MR grade $\geq 2$ of $95 \%$ and $76 \%$, respectively.

\section{Is the RMA still an option?}

The results reported by Furukawa et al. ${ }^{1)}$ seem to coincide with those reported by Kainuma et al. ${ }^{9)}$ The evidence suggests that the patients with smaller ventricular chambers and improvement of LV remodeling have good results in the long run even with the use of RMA alone because the interpapillary muscle distance (IPMD) is reduced with favorable LV remodeling. Recently, the pivotal role of IPMD has been shown in a report from the Osaka Rosai Hospital. ${ }^{9)}$ The authors clarified the association between LV function, severity of MR, and leaflet tethering parameters after RMA. In all, 44 patients who underwent isolated RMA, between 2004 and 2015 were studied. During a median follow-up period of 66 months, the LV function, anterior and posterior PM tethering distance, anterior leaflet angle, and IPMD improved for 33 patients. Change in IPMD (31 \pm 6 to $25 \pm 5 \mathrm{~mm}$ ) and posterior PM tethering ( $37 \pm 4$ to $32 \pm 4 \mathrm{~mm}$ ) were independently associated with a reduced risk of MR recurrence (parameter estimate of 0.299 , standard error of mean (SEM) 0.110; $\mathrm{P}=.013$ and parameter estimate of -0.104 SEM 0.045; $\mathrm{P}=.035$ ). Moreover, the IPMD change was independently associated with a change in LV end-systolic dimensions (parameter estimate of 0.299 with SEM 0.110; $\mathrm{P}=.013$ ) resulting in a better improvement of IPMD that is linked with favorable reverse remodeling.

RMA causes a temporary improvement in MR when the LVEDD is $<65 \mathrm{~mm}$ and LVESD is $<55 \mathrm{~mm}^{2,3,10)} \mathrm{In}$ both PMA $^{5,6)}(\mathrm{n}=33)$ and The Cardiothoracic Surgical Trials Network trial ${ }^{3)}(n=77)$, patients with severe IMR who did not experience persistent or recurrent MR after RMA had a dramatically smaller LV at 2-year follow-up compared to patients with recurrent MR after RMA alone (PMA LVEDD $52.2 \pm 4.1$ vs $60.4 \pm 2.1$; LVESD $44.2 \pm 3.6$ vs $50.8 \pm 2.5$ and CTSN $43 \pm 26 \mathrm{~mL} / \mathrm{m} 2$ vs $63 \pm 27 \mathrm{~mL} / \mathrm{m}^{2}$ ).

\section{Limitation of PMA procedure}

Surgery of papillary muscles may be appropriate in patients with dilated a LV, with large areas of scar tissue formation, dyskinesia, or a basal aneurysm. ${ }^{2,3,11)}$ However, 
prospective trials on the use of subvalvular surgery of mitral valve are currently insufficient to highlight improvements in postoperative tethering in patients with LV lateral wall dysfunction, persistent LV dyskinesis, severe alteration of LV sphericity, and compromised RV function. ${ }^{1)}$ The concern is related to the predominant lateral displacement of both leaflets due to symmetric tethering that may occur in young patients with idiopathic dilated or ischemic cardiomyopathy (DCM) leading to poorer long term result. ${ }^{1-3,5,6,11)}$

\section{The Role of the RV}

Furukawa et al. ${ }^{1}{ }^{1}$ raise some concerns with regard to RVFAC as a risk factor for cardiac-related mortality. It is the only significant predictor of cardiac-related mortality in univariate analysis (risk ratio $[\mathrm{RR}]=0.88,95 \% \mathrm{CI}$ : $0.78-0.97, \mathrm{P}<0.01)$ and multivariable analysis $(\mathrm{RR}=$ $0.89,95 \%$ CI: $0.76-0.98, \mathrm{P}=0.02$ ).

The absence of revascularization of the RV or the persistence of functional tricuspid regurgitation may determine the evolution toward biventricular dysfunction in cases of adverse ventricular remodeling and persistent MR which affects the functionality of the tricuspid valve in patients who did not undergo tricuspid valve surgery. ${ }^{5)}$ Revascularization and restoration of myocardial kinesis reduce acute distortions of the mitral valve, thus counteracting $\mathrm{LV}$ remodeling, a predictor of poor prognosis among patients with persistent ischemic myocardial disease. ${ }^{2,3)}$ The major presence of scar tissue formation limits the effort of reverse remodeling, which is associated with improved outcomes. ${ }^{3,6)}$ Therefore, the combined procedure of CABG with the concomitant valve and SVR is suitable to avoid the risk of MR recurrence. Patients can benefit from tricuspid annuloplasty to avoid progression to RV dysfunction. ${ }^{5)}$

\section{Conclusion}

IMR is a complex condition as highlighted by the multitude of surgical interventions available. We have not even considered the role of medical management and surgical experience in the management of IMR which are out with the remit of this study. However, Furukawa and colleagues have highlighted further benchmarks to consider such as the RVFAC. Further studies are needed to highlight the role of RMA \pm SVR in the long run ( $>10$ years), guided by imaging findings to monitor LV remodeling alongside biventricular function to provide clarity.

\section{Disclosure Statement}

None.

\section{References}

1) Furukawa $K$, Yano $M$, Sakaguchi $S$, et al. Clinical outcomes of a customized mitral valve plasty for functional mitral regurgitation with a low ejection fraction and implications for preoperative right ventricular function. Ann Thorac Cardiovasc Surg 2020; Jun 10. doi 10.5761/atcs.oa.20-00035.Online ahead of print.

2) Nappi F, Lusini M, Avtaar Singh SS, et al. Risk of ischemic mitral regurgitation recurrence after combined valvular and subvalvular repair. Ann Thorac Surg 2019; 108: 536-43.

3) Michler RE. Learning from controversy: management of severe ischemic mitral regurgitation at the time of CABG. Ann Thorac Surg 2019; 108: 321-23.

4) Nappi F, Spadaccio C, Fraldi M. Reply: papillary muscle approximation is an anatomically correct repair for ischemic mitral regurgitation. J Am Coll Cardiol 2016; 68: $1147-8$.

5) Nappi F, Lusini M, Spadaccio C, et al. Papillary muscle approximation versus restrictive annuloplasty alone for severe ischemic mitral regurgitation. J Am Coll Cardiol 2016; 67: 2334-46.

6) Nappi F, Spadaccio C, Nenna A, et al. Is subvalvular repair worthwhile in severe ischemic mitral regurgitation? Subanalysis of the Papillary Muscle Approximation trial. J Thorac Cardiovasc Surg 2017; 153: 286-95.e2.

7) Bothe W, Timek TA, Tibayan FA, et al. Characterization of 3-dimensional papillary muscle displacement in in vivo ovine models of ischemic/functional mitral regurgitation. J Thorac Cardiovasc Surg 2019; 157: 1444-49.

8) Nappi F, Nenna A, Spadaccio C, et al. Predictive factors of long-term results following valve repair in ischemic mitral valve prolapse. Int J Cardiol 2016; 204: 218-28.

9) Kainuma S, Funatsu T, Kondoh $\mathrm{H}$, et al. Beneficial effects of restrictive annuloplasty on subvalvular geometry in patients with functional mitral regurgitation and advanced cardiomyopathy. J Thorac Cardiovasc Surg 2018; 156: 630-38.e1.

10) Nappi F, Spadaccio C, Chello M, et al. Double row of overlapping sutures for downsizing annuloplasty decreases the risk of residual regurgitation in ischaemic mitral valve repair. Eur J Cardiothorac Surg 2016; 49: 1182-7.

11) Nappi F, Avtaar Singh SS, Padala M, et al. The choice of treatment in ischemic mitral regurgitation with reduced left ventricular function. Ann Thorac Surg 2019; 108: 1901-12. 